\title{
Fractured 3D Object Restoration and Completion
}

\author{
A. Andreadis, R. Gregor, I. Sipiran ${ }^{\dagger}$ P. Mavridis, G. Papaioannou, T. Schreck ${ }^{\dagger}$
}


Figure 1: We introduce a pipeline for restoration of 3D shapes based on reassembly of fragments and completion of missing parts. First results show the effectiveness of the approach for restoration of real-world fragment data from the Cultural Heritage domain.

\section{Introduction}

The problem of object restoration from eroded fragments where large parts could be missing is of high relevance in archaeology. Manual restoration is possible and common in practice but it is a tedious and error-prone process, which does not scale well. Solutions for specific parts of the problem have been proposed but a complete reassembly and repair pipeline is absent from the bibliography. We propose a shape restoration pipeline consisting of appropriate methods for automatic fragment reassembly and shape completion. We demonstrate the effectiveness of our approach using real-world fractured objects.

\section{Reassembly and Repair Workflow}

We extend, instantiate and apply our preliminary restoration pipeline described earlier in [Gregor et al. 2014] as follows:

1. Fracture Surface Classification Step. First, we segment the input fragments using a region-growing approach and perform a classification step based on local descriptors, in order to conservatively identify potentially fractured areas.

2. Pairwise Alignment Step. Fragments are matched pairwise by an appropriate contact surface metric [Mavridis et al. 2015], while feature curves spanning across the intact surfaces of multiple fragments are utilized to address the problems that are hard to solve using contact surfaces (heavy erosion or large missing parts).

3. Multi-part Reassembly step. Based on pairwise alignments of fragments, we reassemble objects using Kruskal's algorithm in a graph approach and using penetration tests and edge discards we ensure penetration-free only reassemblies. Manual constraints are also supported. Solutions are refined using an iterative process similar to bundle adjustment. After fragment reassembly, formed clusters are frequently still incomplete, due to missing or unmatched fragments, or erosion, a case that is handled in the next steps.

\footnotetext{
*Athens University of Economics and Business

${ }^{\dagger}$ University of Konstanz
}

4. Completion Based on Shape Symmetry. Alternatively, we also use symmetry when possible to complete objects. In order to find the symmetry plane candidates, we use a voting scheme based on local features [Sipiran et al. 2014].

5. Completion Based on Template Shapes. In this step, identifying missing parts is formulated as a partial retrieval problem. We query a database of template shapes (e.g. randomized instances of parametric surfaces, digitized $\mathrm{CH}$ objects or CAD models) by the set of assembled fragments. We adapt [Bronstein and Bronstein 2011] by computing a second descriptor that incorporates a weighting scheme based on the surface classification. We compare both query descriptors to each template descriptor by a bipolar distance function.

6. Finishing Step. Finally, the reassembled and completed fragments are merged to form a final complete object. Remaining minor shape defects can be interactively finished by smoothing and small-scale inpainting operations using an automation of the userdriven [Takayama et al. 2011]. Missing parts may be exported for $3 \mathrm{D}$ printing and completion of real objects. First restoration results using real archaeological fragment data are promising.

These restoration steps can be flexibly combined to serve specific restoration tasks. First results of application on real-world fragment data from the Archaeology domain are promising (see Figure 1).

\section{References}

Bronstein, A., And Bronstein, M. 2011. Shape google: Geometric words and expressions for invariant shape retrieval. ACM Transactions on Graphics.

Gregor, R., Sipiran, I., Papaioannou, G., Schreck, T., AnDREADIS, A., AND MAVRIDIS, P. 2014. Towards automated 3D reconstruction of defective cultural heritage objects. In Ws. on Graphics and Cultural Heritage, EG, 135-144.

Mavridis, P., Andreadis, A., ANd Papaioannou, G. 2015. Fractured Object Reassembly via Robust Surface Registration. In EG 2015 - Short Papers, The Eurographics Association.

Sipiran, I., Gregor, R., AND Schreck, T. 2014. Approximate symmetry detection in partial 3D meshes. Computer Graphics Forum 33, 7, 131-140.

TAKayama, K., Schmidt, R., Singh, K., Igarashi, T., Boubekeur, T., AND SoRKInE, O. 2011. Geobrush: Interactive mesh geometry cloning. Computer Graphics Forum, 30, 613-622. 